\title{
BMJ Open Economic evaluations of clinical pharmacy services in China: a systematic review
}

\author{
Xue Mi, ${ }^{1}$ Xuelong Su, ${ }^{2}$ Ziyan Jin, ${ }^{3}$ Linan Zeng, ${ }^{4}$ Zhuo Li, ${ }^{1}$ Xiaowen Wang, \\ Peng Zhang, ${ }^{1}$ Yan Zuo (i) ${ }^{1}$
}

To cite: Mi X, Su X, Jin Z, et al. Economic evaluations of clinical pharmacy services in China: a systematic review. BMJ Open 2020;10:e034862. doi:10.1136/ bmjopen-2019-034862

- Prepublication history and additional material for this paper are available online. To view these files, please visit the journal online (http://dx.doi. org/10.1136/bmjopen-2019034862).

$\mathrm{XM}$ and $\mathrm{XS}$ are joint first authors.

Received 13 0ctober 2019 Revised 21 October 2019 Accepted 22 0ctober 2019

Check for updates

(C) Author(s) (or their employer(s)) 2020. Re-use permitted under CC BY-NC. No commercial re-use. See rights and permissions. Published by BMJ.

${ }^{1}$ Department of Pharmacy, Shaanxi Provincial People's Hospital, Xi'an, China

${ }^{2}$ Dept. of Pediatric Dentistry, College of Stomatology, Xi'an Jiaotong University, Xi'an, China ${ }^{3}$ West China School of Pharmacy, Sichuan University, Chengdu, Sichuan, China ${ }^{4}$ Department of Pharmacy, West China Second University Hospital, Sichuan University, Chengdu, China

Correspondence to

Yan Zuo; mixue0409@163.com

\section{ABSTRACT}

Objective This article reviewed research conducted on economic evaluations of clinical pharmacy services (CPS) in China. We aimed to identify the types of CPS and the possible economic effects of these services and to hopefully provide some suggestions for designing future economic evaluations of pharmacy interventions in the region.

Design Systematic review.

Data sources Several English databases (PubMed, Embase, The Cochrane Library, National Health Service Economic Evaluation Database), Chinese databases (China National Knowledge Infrastructure, VIP, Chinese Biomedical Literature Database and WanFang Data) and search engines (Google Scholar and BaiDu Scholar) were searched through December 2017.

Eligibility criteria for selecting studies Studies with an economic assessment of CPSs in China were included.

Data extraction and synthesis Two reviewers independently screened the studies, extracted the data, assessed the quality of the included studies and then qualitatively analysed the results.

Results Forty articles were included in the final analysis. Most studies were performed in hospitals and the intervention populations mainly included adults. The types of pharmaceutical services included antimicrobial management, chronic disease state management and multidimensional clinical pharmaceutical services. A positive economic benefit associated with CPS was noted in $80 \%(n=32)$ of these articles, showing that CPS were associated with cost savings and improved patient outcomes. However, only three studies were full economic evaluations, using the method of cost-effectiveness analysis.

Conclusion CPS was associated with cost savings and generated positive economic value. With the expanding role of pharmacists in the healthcare sector, it is suggested that new pharmaceutical services be used in future studies and that high-quality full economic evaluations capturing both expenses and cost savings be conducted.

\section{INTRODUCTION}

The concept and practice of clinical pharmacy services (CPS) originated in the USA. ${ }^{1}$ The American College of Clinical Pharmacy's (ACCP) definition of clinical pharmacy refers to the contribution that pharmacists make

\section{Strengths and limitations of this study}

This study is the first to review economic evaluation studies on clinical pharmacy services in China by using a formal systematic review approach.

- This study was based on an evidence-based methodology to provide evidence to healthcare decision makers regarding the economic value of clinical pharmacy services, and it provides some suggestions and references for designing future economic evaluations of pharmacy interventions in the region.

- The possible risk of bias in the included studies was assessed using the American College of Clinical Pharmacy checklist that was designed specifically to grade the quality of economic methods, the Cochrane Collaboration Methodology or the Newcastle-0ttawa Scale.

- However, the heterogeneity among the included trials was relatively high, so we analysed the results qualitatively.

in applying their professional knowledge to ensure high-quality rational drug therapy to produce optimal patient outcomes. ${ }^{2}$ The published literature suggests that CPS could improve patient clinical outcomes, as pharmacists use their skills and knowledge to make the best use of medications in order to ensure safe and effective pharmacotherapy. ${ }^{34}$ Pharmacist participation in physician rounds and provision of medication guidance can reduce the frequency of adverse drug events (ADEs) and medication errors and improve medication adherence. ${ }^{5}$ In addition to clinical benefits, patients' physical, emotional, functional and social well-being have also been positively affected when measured using health-related quality-of-life assessments. ${ }^{6}$

In 2009, wide-ranging reforms of the healthcare system were announced in China. Hospitals account for approximately threequarters of pharmaceutical sales in China. To compensate for diminishing state contributions, hospitals were permitted to charge a mark-up of up to $15 \%$ on medicines. The 
mark-up has had serious consequences. Doctors' salaries and bonuses were linked to drug sales, spurring excessive and inappropriate prescriptions. ${ }^{7}$ In 2009, this system of mark-ups was abolished, and billions of yuan were poured into the public system, which aimed to control China's increasing health expenditure to provide affordable healthcare. ${ }^{7}$ However, healthcare costs have continued to rise in China, which means that healthcare institutions need to identify and adopt efficient ways to control these costs. Although CPS have been developed in China and play an important role in improving patients' clinical outcomes, faced with limited resources and skyrocketing healthcare costs, healthcare policy-makers haven grown increasingly focused on the economic effectiveness and cost of services.

Economic evaluations play an important role in informing resource allocation decision and lead to the provision of information to public policy-makers and healthcare payers about the good value for the invested money afforded by a healthcare programme. ${ }^{8}$ Published evidence of the economic value of CPS is an important resource, which can be used to justify pharmacist-led programmes and can also improve net revenue by reducing medical expenses. Beyond that, economic evaluations can also help improve the quality of CPS by guiding the choice of the most effective and cost-effective pharmacy programmes. ${ }^{9-11}$

China became interested in CPS as early as 1962; however, due to the economic and political climate, CPS were finally developed in the 21st century. While still in the early stages of development, CPS are becoming firmly established with the support of China's Ministry of Health $(\mathrm{MoH})$ of the People's Republic of China. ${ }^{12}$ In 2011, the $\mathrm{MoH}$ issued a policy that all secondary and tertiary hospitals should have at least three and five full-time clinical pharmacists, respectively. Furthermore, pharmacists needed to be trained in infectious diseases to be able to give guidance and approval for restricted antimicrobial use. ${ }^{13}$ In 2018, the MoH indicated that pharmacists have the ultimate responsibility for prescription review. ${ }^{14}$ The role of the pharmacist is becoming increasingly important, but whether and how to 'pay pharmacists for patient care' is still an area of debate.

In China, many significant original studies have been published about the measurement of the economic impact of CPS. To date, no study has reviewed economic evaluation studies on CPS in China using a formal systematic review approach. The objective of this study was to review these studies, to identify the types of CPS and the possible economic effects of these services in China, and to provide evidence of the economic value of CPS to healthcare decision makers. Additionally, this study provides some suggestions and references for designing future economic evaluations of pharmacy interventions in the region.

\section{METHOD}

This study was largely based on the Preferred Reporting Items for Systematic Reviews and Meta-Analyses (PRISMA) guidelines for reporting systematic reviews. ${ }^{15}$ The PRISMA checklist is shown in the online supplementary file.

\section{Inclusion criteria}

Participants

Any patient group and the associated prescriptions were included.

\section{Intervention}

All CPS performed by a pharmacist or team of pharmacists were considered interventions. In this review, the term intervention conforms to the unabridged ACCP definition of a clinical pharmaceutical intervention, in which pharmacists provide patient care that optimises medication therapy and disease prevention and promotes health. ${ }^{2}$

\section{Outcomes}

The primary outcome was a full or partial economic assessment of the cost to provide a service. A full economic evaluation was conducted using four techniques: cost-minimisation analysis, cost-effectiveness analysis, cost-utility analysis and cost-benefit analysis. Partial economic evaluations were considered in terms of costs and consequences. ${ }^{16}$

Study design

Randomised controlled trials, semirandomised controlled trials, non-randomised controlled trials, cohort studies and before-and-after studies were included.

Setting

The research sites were in China.

\section{Language}

The articles were published in English or Chinese.

\section{Exclusion criteria}

Studies were excluded that evaluated only humanistic or clinical outcomes without an economic assessment; studies published in abstract form only or unoriginal work (letters or reviews) were also excluded. When full texts of the studies were unavailable, they were also excluded, and repeated publications were excluded.

\section{Search strategy}

Relevant studies were identified by electronically searching the following databases: English databases (PubMed, Embase, the Cochrane Library, National Health Service Economic Evaluation Database), Chinese databases (China National Knowledge Infrastructure, WanFang Database, Chinese Biomedical Literature Database, VIP Database for Chinese Technical Periodicals) and search engines (Google Scholar and Bai Du Scholar). Articles were retrieved from the date of the inception of the database to 24 December 2017. In addition, the bibliographies of relevant identified articles were manually searched. The search terms combined the Medical Subject Headings (MeSH) headings 
with free-text words, including 'clinical pharmacy', 'pharmaceutical services', 'pharmaceutical care', 'pharmacy service', 'pharmacist', 'economic evaluation', 'cost-utility analysis', 'cost-benefit analysis' and 'cost-effectiveness analysis'. More details about the search strategy can be found in the attachment (shown in online supplementary file).

\section{Study selection and data extraction}

Two study investigators independently screened and crosschecked all articles and extracted the data. In instances of disagreement, two reviewers resolved their differences of opinion by discussion to reach a consensus or consulted a third independent investigator to reach a consensus. Study selection was based on titles and abstracts, and the full texts were read after excluding obviously unrelated studies to make the ultimate decision regarding whether the study would be included. The extracted data included: the study design, setting, population, sample size, type of intervention, type of economic evaluation and economic outcomes.

\section{Study appraisal and analysis}

The assessment of study quality was performed with the checklist from the ACCP that was designed specifically to grade the quality of the economic methods used, which included three assessment items: (1) the description of the comparator group used; (2) the evaluation and description of the programme costs; and (3) the evaluation and description of the outcomes. ${ }^{9}$ Studies were considered 'good quality' when they met all three criteria. Studies that lacked a comparator or had multiple or fatal flaws were determined to be 'poor quality'. All other studies were labelled 'fair quality' if they only contained an evaluation and description of the economic outcomes. In addition, we assessed the risk of bias in the included studies. The included randomised controlled trials (RCTs) were assessed using the Cochrane risk-of-bias criteria. ${ }^{17}$ The cohort and before-and-after studies were assessed using the Newcastle-Ottawa Scale (NOS), which uses the semiquantisation star system with a full score of 9 stars, and the content evaluated includes the study selection, comparability and outcome. On the NOS, $\geq 7$ stars indicates good quality, 5-7 stars indicates intermediate quality and $\leq 4$ stars indicates poor quality. ${ }^{18}$

The heterogeneity of the results prevented a combined statistical analysis, so a qualitative analysis was carried out in this review to classify these articles by the type of CPS, setting and different perspectives, and to analyse the economic impact, existing research status and problems.

\section{Patients and public involvement statement}

Patients and the public were not involved in this study.

\section{RESULTS}

A total of 2438 relevant papers were obtained from the search, of which 608 were excluded because they were duplicates. Based on the abstracts, 1764 were excluded because they were irrelevant $(n=792)$, did not include

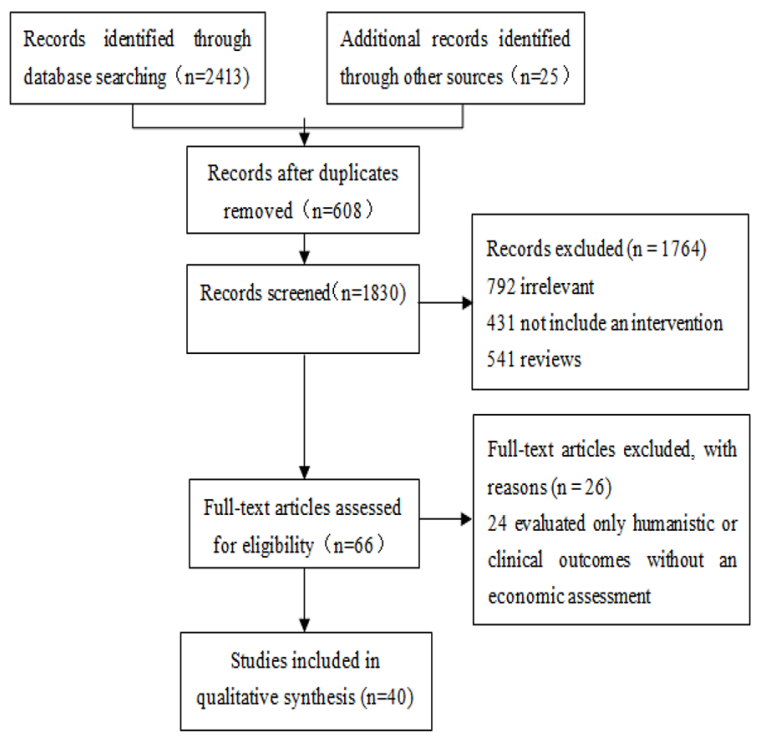

Figure 1 Literature search method and screening results.

an intervention $(n=431)$ or were reviews $(n=541)$. Of the remaining 66 articles, 26 articles were excluded from the review because they evaluated only humanistic or clinical outcomes without an economic assessment. A total of 40 studies met the inclusion criteria for the review. The screening process and results are shown in figure 1.

\section{Basic characteristics and risk of bias in included studies}

The number of studies on this topic has gradually increased since 2010. The study designs were divided into RCTs $(n=13)$, cohort studies $(n=16)$ and before-and-after intervention studies $(\mathrm{n}=11)$. Among the included studies, $38(95 \%)$ were conducted in hospitals, while the others were performed in clinics or community pharmacies. The research population mainly focused on adults, and only one study focused entirely on a paediatric population. All of these studies had a concurrent or historical control and were conducted from the perspective of the hospital. Four studies $(10 \%)$ were deemed to be 'good quality', while the others were described as being 'fair quality' as they only evaluated and described the economic outcomes, such as the drug or hospitalisation cost savings. The characteristics of the 40 included studies are summarised in table 1 .

The results of the assessment of the risk of bias in the RCTs are shown in table 2. The bias of risk with regard to the 'blinding of participants and personnel (performance bias)' and the 'blinding of outcome assessment (detection bias)' were high in the included RCTs (because the pharmacist must interact with the patient face to face to provide give drug-use guidance according to each patient's condition). In addition, 'random sequence generation (selection bias)' and 'allocation concealment (selection bias)' had high levels of the risk of bias in almost all the included RCTs; they reported that a randomisation method was employed but do not clearly describe it. The risk-of-bias assessments in the cohort and before-and-after studies showed that $18.5 \%$ $(n=5)$ of the studies were 'good quality', while the others $(\mathrm{n}=22,81.5 \%)$ were 'intermediate quality'. The results of 


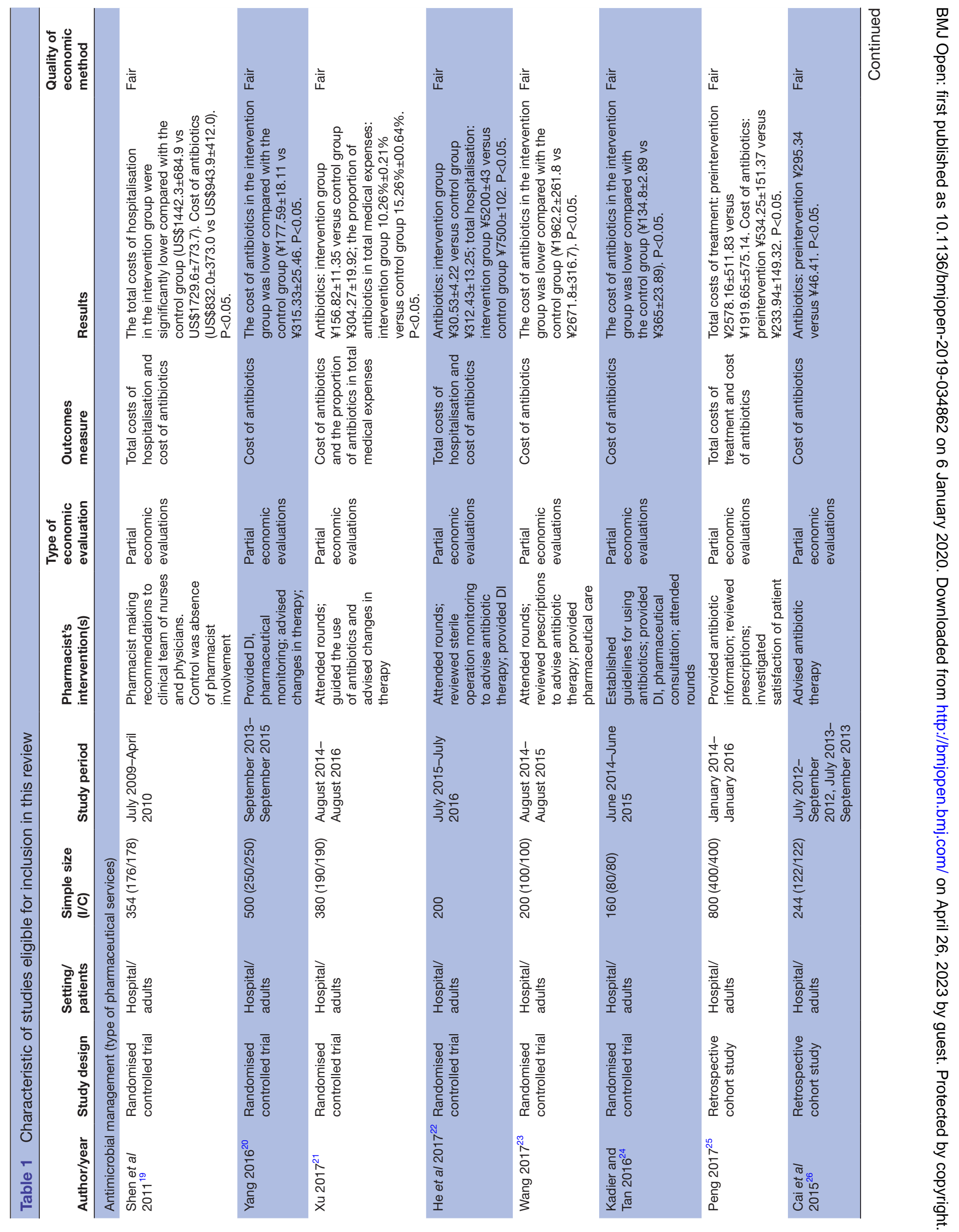




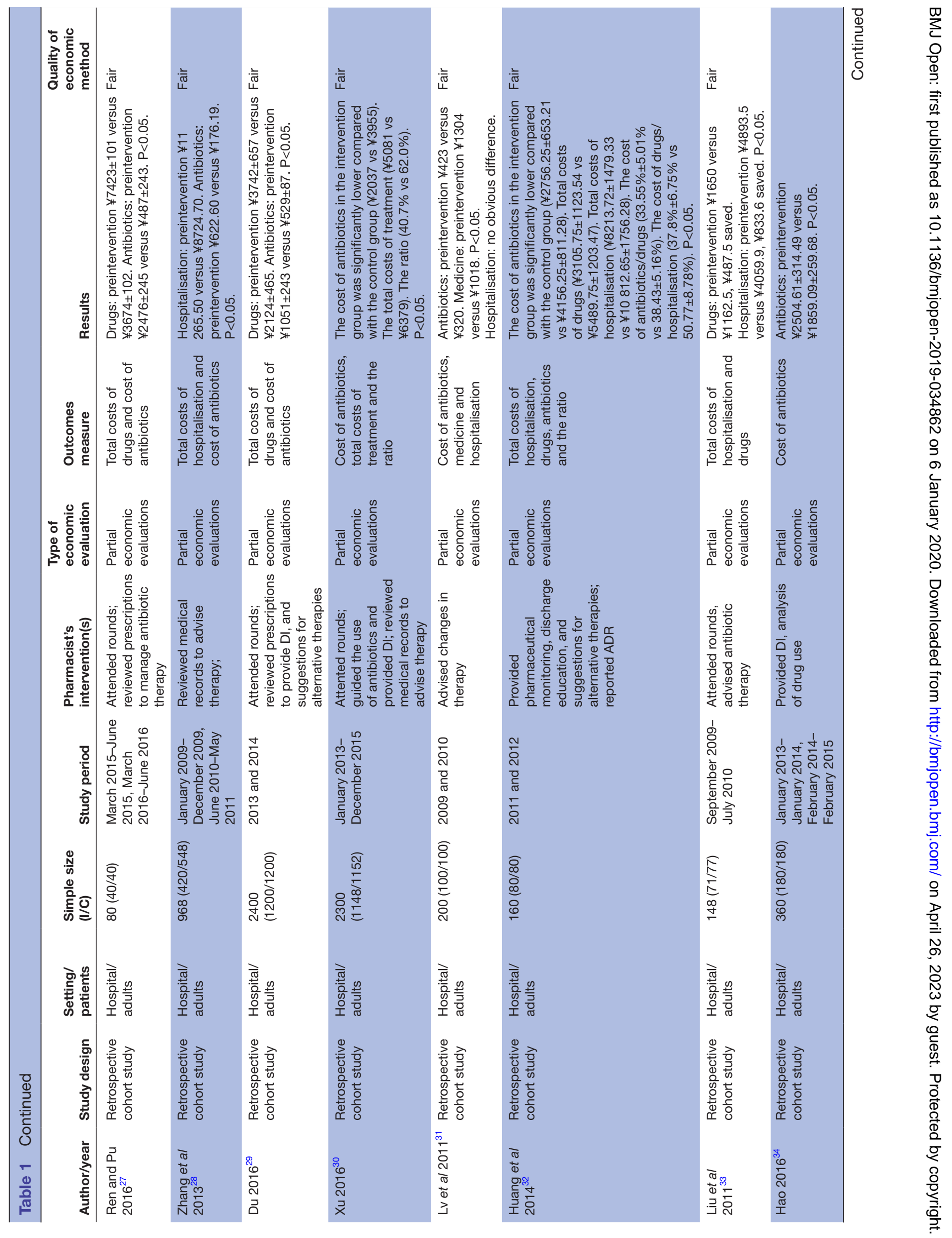




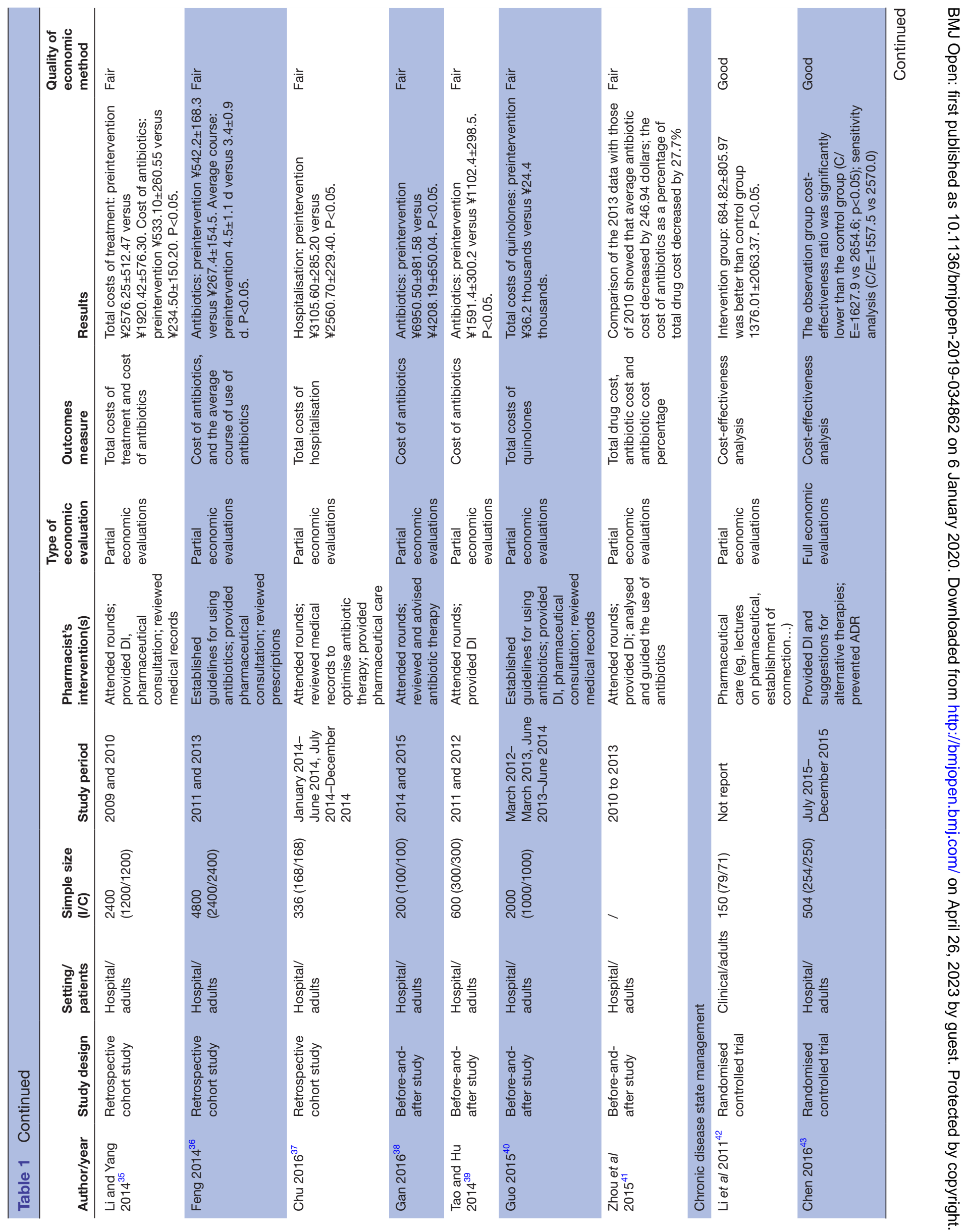




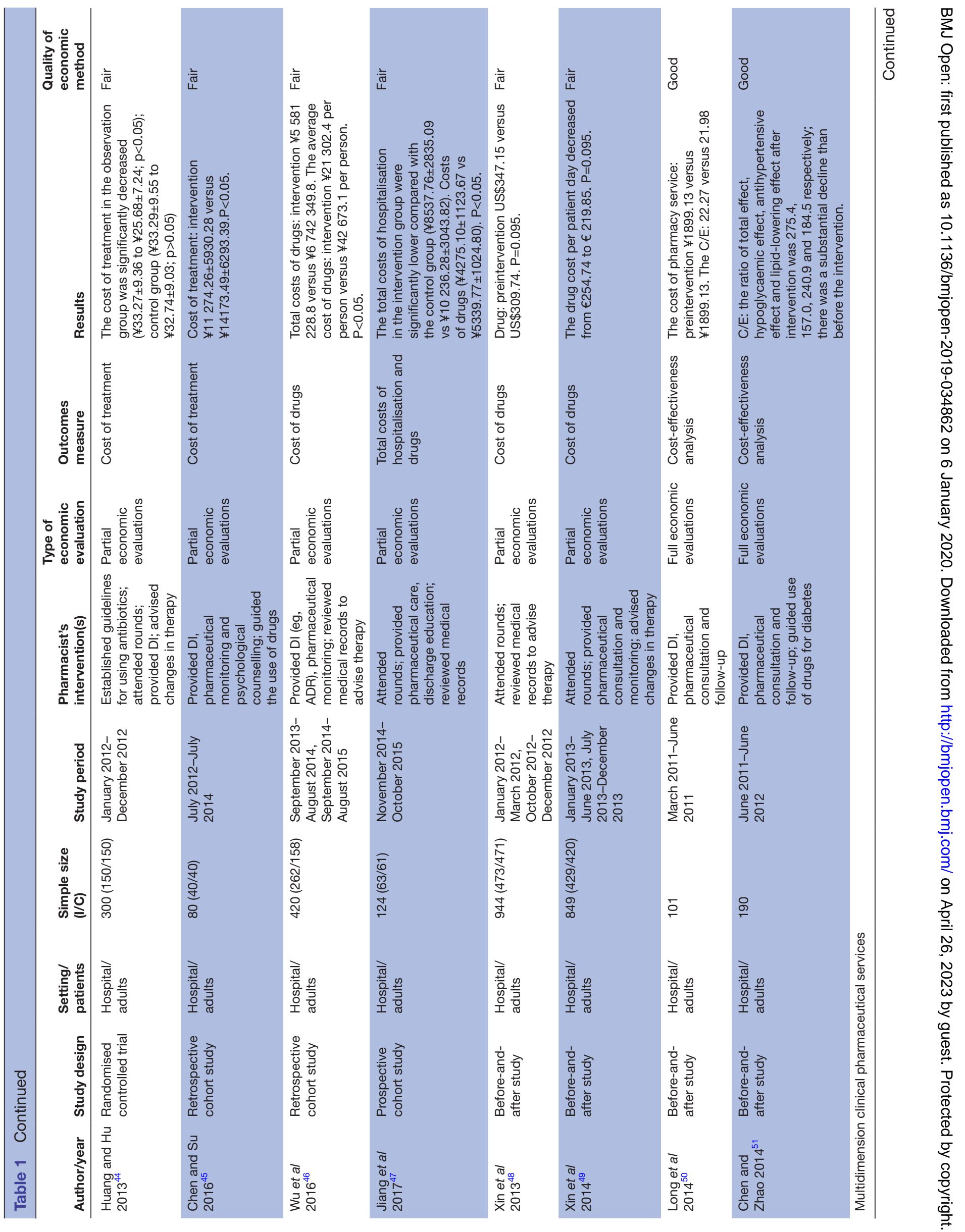




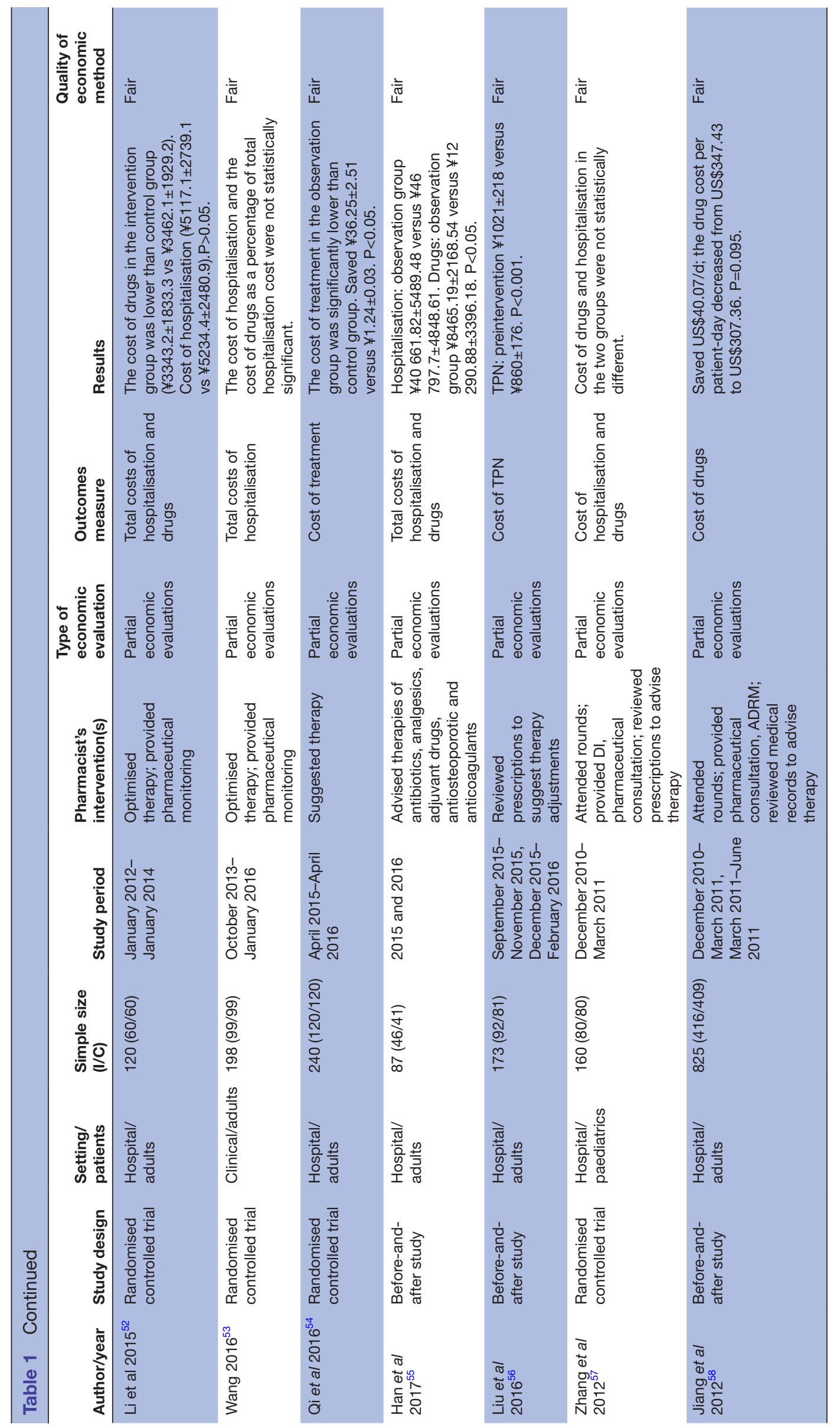


Table 2 Risk of bias for randomised controlled trials

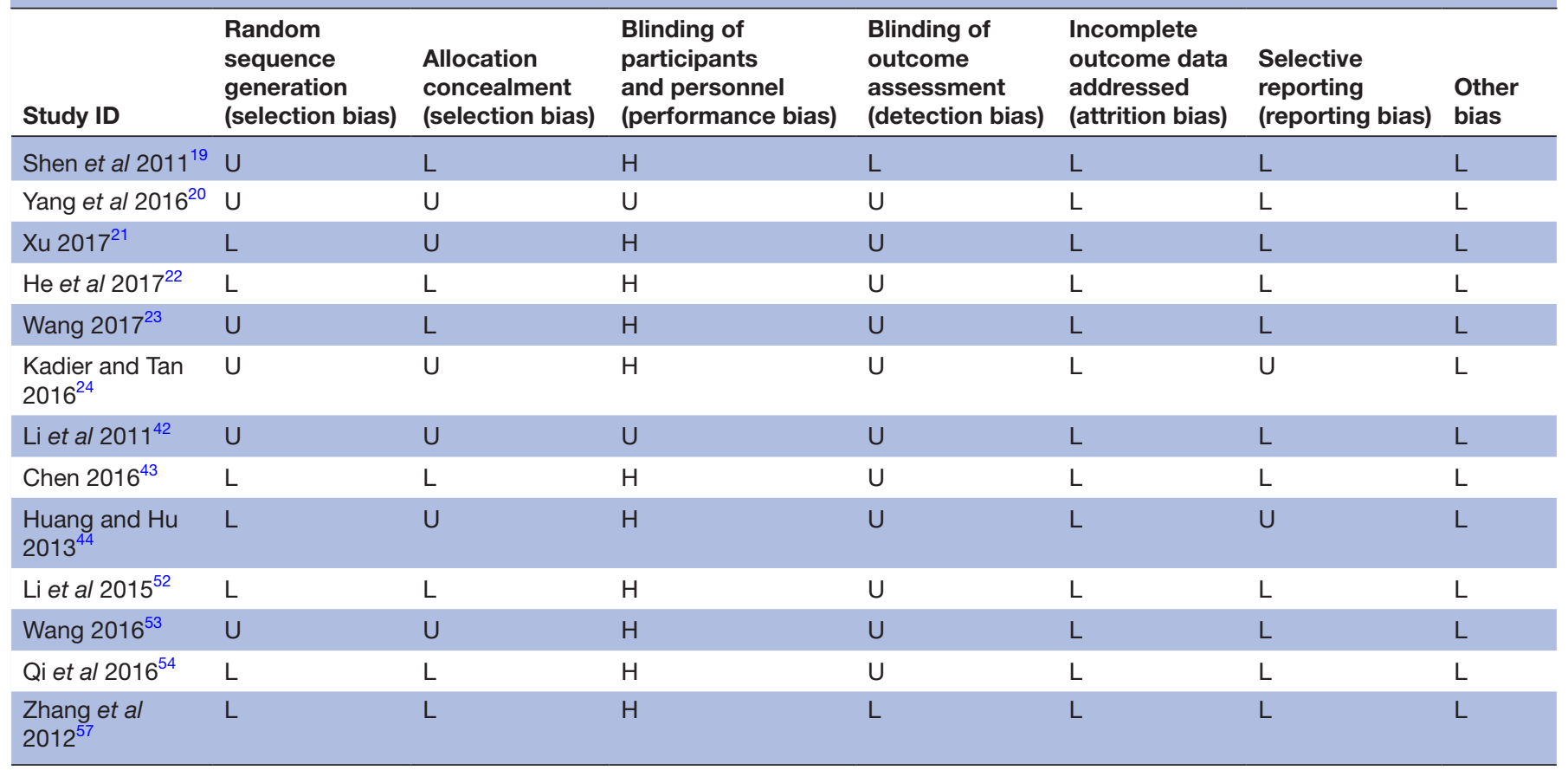

$\mathrm{H}$, high risk;ID, identification; L, low risk; $U$, unknown.

risk-of-bias assessments in the cohort and before-and-after studies are shown in table 3 .

\section{Clinical pharmacy interventions}

The type of CPS was classified as one of the three following areas: (1) antimicrobial management services ${ }^{19-41}$ (23 $(57.5 \%))$ that primarily focused on antimicrobial use and may have included predefined guidelines for the provision of dosing recommendations or the preferred drug; (2) chronic disease state management services ${ }^{42-51}$ $(10(25 \%))$ that were primarily directed at patients with a specific disease state or diagnosis, such as a diabetes management programme; and (3) multidimensional clinical pharmaceutical services ${ }^{52-58}(7(17.5 \%))$ that encompassed a broad range of activities based on the need of patients. The content of the interventions included the adjustment of dosages; the provision of advice regarding therapeutic drug monitoring; the evaluation of drug history; the provision of drug information to physicians and patients to prevent pharmacological and physicochemical interactions, prescribing and transcription errors and ADEs; the participation in physician rounds; and the implementation and tracking of the use of guidelines regarding the correct use of drugs. In these studies, interventions were generally undertaken by clinical pharmacists and did not involve dispensing services.

\section{Economic impact assessment}

Three studies were full economic evaluations, using the method of cost-effectiveness analysis. ${ }^{43} 5051$ Others conducted partial economic evaluations that were limited to the direct healthcare costs, estimating the drug and hospitalisation cost savings only. Most studies $(97.5 \%)$ did not calculate the pharmacist's labour costs. In addition, only two studies (5\%) conducted a sensitivity analysis to account for uncertainty in the estimates of the costs and consequences,. ${ }^{43} 51$ An incremental cost-benefit ratio or incremental cost-effectiveness ratio (ICER) was presented in one study. ${ }^{50}$ The transferability of results to other settings or countries was not discussed in all the studies' results.

A positive economic benefit associated with pharmacy services was noted in $80 \%(n=32)$ of these studies. The other studies $(n=8,20 \%)$ also showed lower medical costs due to pharmacist intervention; however, these trials did not find statistically significant differences in the medication costs saved. In the following discussion, we provide a detailed description of these studies categorised by the type of clinical pharmacy intervention.

\section{Economic impact of antimicrobial management}

Twenty-three studies on CPS included antimicrobial management, and the interventions were conducted in hospitals. ${ }^{19-41}$ All of these assessments were partial economic evaluations; among them, 21 studies with a control group applied statistics to compare the two groups, with economic outcomes showing that pharmacists providing antimicrobial management services led to overall cost savings (including the cost of antibacterial drugs, hospitalisation expenses and total treatment costs) in the intervention groups, and the results showed a significant difference $(\mathrm{p}<0.05) .{ }^{19-39}$ Another two studies also showed that antibiotic costs decreased but were not reported as a statistically significantly different. $^{4041}$ 
Economic impact of chronic disease state management

Ten studies described three disease state management programmes (tuberculosis, diabetes and hypertension). ${ }^{42-51}$ Among them, three studies were full economic evaluations, and all of them used cost-effectiveness analyses. $^{45051}$ The pharmacist provision of education to patients with diabetes resulted in a cost-effectiveness ratio (CER) of 21.98 in comparison with the control group (22.27) and the ICER was 21.02, which showed a positive economic outcome based on the estimated cost of providing the service. ${ }^{50}$ A stewardship programme provided for hypertension was investigated, and the study reported a reduction in the CER (pharmacist present 1627.0 vs no pharmacist 2654.6, $\mathrm{p}<0.05$ ) when pharmacists were included as a part of a multidisciplinary team. ${ }^{43}$ Another study showed a substantial decline in the CER compared with before an intervention occurred (intervention 275. 4 vs no intervention 384. 5), and the CPS improved the effect of the treatment on patients with diabetes mellitus in the community, thereby reducing the cost of treatment. ${ }^{51}$ Other studies were partial economic evaluations, and they only reported the cost of treatment (including drug charges and hospitalisation expenses), which were reduced after intervention. Service costs were considered in one study, ${ }^{50}$ but the other studies omitted them.

\section{Economic impact of multidimensional CPS}

There were seven studies on such pharmaceutical services. All of them were partial economic evaluations, which only reported decreased medical costs, and four of the studies showed no statistically significant differences, although medical costs decreased due to pharmaceutical interventions in the results. ${ }^{52535758}$ The cost of providing the service was not stated in all studies.

The detailed economic results of each study are shown in table 1.

\section{DISCUSSION}

In this systematic review, a positive economic benefit associated with CPS was noted in $80 \%(n=32)$ of the articles, showing that CPS were associated with cost savings. The context of CPS has been expanding from medication dispensing towards providing individualised care in China. The types of services include antimicrobial management, chronic disease state management and multidimensional clinical pharmaceutical services, and the interventions are concentrated in hospitals and community clinics. However, compared with studies from other countries, the types of services provided in China and the settings of the interventions show a lack of diversity. For example, an economic assessment of the CPS in the USA by the ACCP showed that types of CPS also included health screening services, laboratory testing services, wellness programmes, immunisation services and medication therapy management. In addition, studies in the USA evaluated interventions in community settings, clinics, long-term monitoring institutions, rehabilitation organisations and so on. ${ }^{39}$ Faced with China's current healthcare reform, increasing numbers of hospitals are paying more attention to the development of CPS with the hope that pharmacists can provide more correct guidance regarding drug use, thereby saving medical resources. By expanding the breadth of CPS, further economic savings could be garnered; however, the evaluation of services in other settings, such as the community, will be needed to determine if similar savings are obtained.

CPS are still in the early stages of development in China, and the number of studies on the economic evaluation of pharmaceutical services has only gradually increased since 2010. Most of the included studies focused on adults. Among the included studies, $80 \%$ reported a positive economic benefit associated with CPS, but only four $(10 \%)$ of the studies were 'good quality'. Most of the studies were partial economic evaluations that cited clinical effects as the main outcome, and the economic outcome was added as an afterthought; these studies only reported direct cost savings, for example, hospitalisation and drug-related cost savings. In addition, these studies did not calculate hidden costs and potential savings; only one study took into account the pharmacist's labour costs and time input. When input costs are not appropriately estimated, it leads to the overestimation of the cost savings, rendering it impossible to make an informed decision regarding the true value of CPS. Future economic evaluations should pay more attention to paediatric populations and conduct full economic evaluations in which all relevant direct healthcare costs and the indirect costs of productivity loss are considered and measured, including pharmacist employment costs.

CPS have existed in China for more than 20 years, and there is a vast body of original research about the economic impact of pharmacy services. However, to the best of our knowledge, the present study is the first to systematically review the economic evaluation literature on CPS in China. The results of this study showed that CPS have resulted in beneficial outcomes for patients, such as reducing drug costs, the length of stay and the cost of treatment; in addition, one pilot programme showed that nearly all directors of hospitals, doctors, pharmacy directors and patients surveyed $(n=207)$ supported the role of clinical pharmacists. ${ }^{59}$ However, whether and how to "pay pharmacists for patient care' is still an area of debate. Our research provides information that can be used by healthcare administrators regarding the potential return on investment afforded by CPS. This information can help guide those responsible for allocating medical resources when faced with reforms of the healthcare system and skyrocketing healthcare costs. This work further highlights the gaps in knowledge where further research is needed, forming the foundation for designing future economic evaluations of pharmacy interventions in the region.

This review has some limitations. First, in the process of study selection, some grey articles were unavailable and not included in this review. Second, this study might be 
affected by publication bias, as negative or non-significant results may have remained unpublished. Third, the literature search was conducted until January 2018; next, we will update the literature review every 3 years. Fourth, the included studies were conducted in different hospitals, with different kinds of patient and various interventions, making it difficult to compare the results and preventing the performance of a meta-analysis. Therefore, we qualitatively analysed the results. In addition, most included studies were partial economic evaluations and had a risk of bias. Among the included RCTs, $46.2 \%(\mathrm{n}=6)$ had selection bias (random sequence generation and allocation concealment). Many articles reported that they used randomisation methods; however, some of them did not clearly report the randomisation approach, which may reduce the credibility of the evaluation findings. Furthermore, sensitivity analyses were not considered in many studies because the changes in parameter values could result in different results and conclusions. All cohort studies used retrospective experimental designs with the recommended methods for collecting and reporting economic information. Future studies should adopt full economic evaluations in which all relevant costs and outcomes are considered and measured. Furthermore, efforts should be made to improve the quality of the studies to decrease the risk of bias. All guidelines available regarding the design and reporting of economic evaluations should be used by authors when developing the economic portion of their studies. ${ }^{60}$

\section{CONCLUSION}

Clinical pharmacy interventions are associated with cost savings. However, most studies had limitations in their methodological quality and applicability to current practice. Compared with CPS and the evaluations of CPS in other countries, the types of pharmaceutical services in China were limited, and most studies adopted partial economic evaluations. It is suggested that new pharmaceutical services be included in future studies and that full economic evaluations capturing both expenses and cost savings be conducted to promote the development of pharmaceutical care.

Contributors XM, XS and LZ performed research design. XM, XS and ZL performed research execution. XM, XS, XW and $\mathrm{PZ}$ performed data analysis. $\mathrm{XM}, \mathrm{XS}$ and $\mathrm{YZ}$ performed manuscript preparation.

Funding This work was supported by the Department of science and technology of Shaanxi Province [2019SF-188] and the Shaanxi Provincial People's Hospital [2016YX-11]

Competing interests None declared.

Patient consent for publication Not required.

Provenance and peer review Not commissioned; externally peer reviewed.

Data availability statement № data are available.

Open access This is an open access article distributed in accordance with the Creative Commons Attribution Non Commercial (CC BY-NC 4.0) license, which permits others to distribute, remix, adapt, build upon this work non-commercially, and license their derivative works on different terms, provided the original work is properly cited, appropriate credit is given, any changes made indicated, and the use is non-commercial. See: http://creativecommons.org/licenses/by-nc/4.0/.
ORCID iD

Yan Zuo http://orcid.org/0000-0001-8607-9626

\section{REFERENCES}

1 Hepler CD, Strand LM. Opportunities and responsibilities in pharmaceutical care. Am J Hosp Pharm 1990;47:533-43.

2 Pharmacy A COC, American College of Clinical Pharmacy. The definition of clinical pharmacy. Pharmacotherapy 2008;28:816-7.

3 Touchette DR, Doloresco F, Suda KJ, et al. Economic evaluations of clinical pharmacy services: 2006-2010. Pharmacotherapy 2014;34:771-93.

4 Nicolas A, Eickhoff C, Griese N, et al. Drug-Related problems in prescribed medicines in Germany at the time of dispensing. Int J Clin Pharm 2013;35:476-82.

5 De Rijdt T, Willems L, Simoens S. Economic effects of clinical pharmacy interventions: a literature review. Am J Health Syst Pharm 2008;65:1161-72.

6 Pickard AS, Hung S-Y. An update on evidence of clinical pharmacy services' impact on health-related quality of life. Ann Pharmacother 2006;40:1623-34.

7 Burki T. China faces challenges to fix its pharmaceutical system. The Lancet 2017;389:353-4.

8 Perraudin C, Bugnon O, Pelletier-Fleury N. Expanding professional pharmacy services in European community setting: is it costeffective? A systematic review for health policy considerations. Health Policy 2016;120:1350-62.

9 Perez A, Doloresco F, Hoffman JM, et al. Economic evaluations of clinical pharmacy services: 2001-2005. Pharmacotherapy 2009;29:128.

10 Chisholm-Burns MA, Graff Zivin JS, Lee JK, et al. Economic effects of pharmacists on health outcomes in the United States: a systematic review. Am J Health Syst Pharm 2010;67:1624-34.

11 Schumock GT, Pickard AS. Comparative effectiveness research: relevance and applications to pharmacy. J 2009;66:1278-86.

12 Penm J, Li Y, Zhai S, et al. The impact of clinical pharmacy services in China on the quality use of medicines: a systematic review in context of China's current healthcare reform. Health Policy Plan 2014;29:849-72.

13 Ministry of Health of the People's Republic of China. Policy on pharmacy administration in health care facilities, 2011 (in Chinese). Available: http://www.moh.gov.cn/mohyzs/s3585/201103/51113. shtml [Accessed 26 December 2018].

14 National Health Commission of the People's Republic of China. Standard for prescription review in medical institutions, 2018. Available: http://www.nhc.gov.cn/ [Accessed 26 Dec 2018].

15 Moher D, Liberati A, Tetzlaff J, et al. Preferred reporting items for systematic reviews and meta-analyses: the PRISMA statement. PLoS Med 2009;6:e1000097.

16 Drummond M, O'Brien B, Stoddart G, et al. Methods for the economic evaluations of health care programs. Oup Catalogue 2005;41.

17 Higgins J, Green S. Cochrane Handbook for Systematic Reviews of Interventions [Access the Cochrane Handbook for Systematic Reviews of Interventions], 2011.

18 Wells GA, Shea BJ, O'Connell D, et al. The Newcastle-Ottawa scale (NOS) for assessing the quality of Non-Randomized studies in metaanalysis. Applied Engineering in Agriculture 2012;18:727-34.

19 Shen J, Sun Q, Zhou X, et al. Pharmacist interventions on antibiotic use in inpatients with respiratory tract infections in a Chinese Hospital. Int J Clin Pharm 2011;33:929-33.

20 Yang Y. Significance assessment of clinical rational application of antibiotics and pharmaceutical intervention. Journal of Clinical Medical 2016;3.

21 Xu Y. Pharmacy intervention impact analysis on the rational application of antibiotics. Journal of North Pharmacy 2017;14.

$22 \mathrm{He} \mathrm{Z}$, Li J, You Q, et al. Evaluation analysis of pharmaceutical intervention on perioperative antibiotic use in patients with type I incision. China Modern Medicine 2017;24:143-57.

23 Wang Y. Analysis of the effect of pharmaceutical intervention on the use of antibiotics in respiratory medicine. World Latest Medicine Information 2017;17.

24 Kadier A, Tan X. Influence analysis of pharmaceutical intervention on the clinical rational use of antibiotics. China Continuing Medical Education 2016;8:171-2.

25 Peng M. Influencing factors of irrational use of antibiotics and clinical intervention 2017;35:595-7.

26 Cai J, Chen W, Chen X, et al. Comparative analysis on application of preventive antibiotics to type I incision operation in pharmaceutical intervention. Chinese Pharmaceutical Affairs 2015;29:423-6. 
27 Ren Y, Pu Y. Analysis of pharmaceutical intervention by clinical pharmacists on clinical irrational use of drugs. World Latest Medicine Information 2016;16.

28 Zhang J, Wang R, Wang Q, et al. Effectiveness of Clinical Pharmacists' Intervention on Perioperative Use of Prophylactic Antibiotics in Patients Undergoing Sterile Operation. Evaluation and analysis of drug-use in hospitals of China 2013;13:84-6.

29 Du L. Analysis of the effect of pharmaceutical intervention on clinical rational drug use by clinical pharmacists. Journal of China Prescription Drug 2016;14.

$30 \mathrm{Xu} \mathrm{J}$. The role of clinical pharmacists in the rational use of antibiotics in the respiratory medicine department. Strait Pharmaceutical Journal 2016;28:238-9.

31 Lv C, Liu J, Chen X. Analysis of effect of Prophylatic pharmaceutical intervention in peri-operative stage of Cesareen. Pharmacy Tody 2011;21:120-4.

32 Huang L, Chen T, Chen J. Research of clinical pharmacy intervention for community-acquired pneumonia in general internal medicine. Pharmacy Tody 2014;24:610-2.

33 Liu M, Wen C, Jiang X, et al. Investigation and analysis of preventive use of antibacterials in the perioperative period of breast surgery. Chinese Journal for Clinicians 2011;39:43-4.

$34 \mathrm{Hao} \mathrm{H}$. Analysis of the effect of pharmaceutical intervention on the application of antibiotics in respiratory medicine. World Latest Medicine Information 2016;16.

35 Li L, Yang Z. Pharmaceutical intervention measures and clinical effect observation on rational use of antibacterial drugs. Sichuan Medical Journal 2014;35:141-2.

36 Feng Z. Effect of pharmaceutical intervention on rational use of antibiotics. Journal of North Pharmacy 2014;11.

37 Chu C. Effect of pharmaceutical intervention on rational clinical application of antibiotics and its related value. Contemporary Medicine 2016;22:137-8.

38 Gan L. Rational application of antibiotics and analysis of pharmaceutical intervention methods in respiratory medicine. Clin $\mathrm{J}$ of Clinical Rational Drug Use 2016;9:27-8.

39 Tao Y, Hu L. Analysis of intervention effect in rational application of clinical antibacterial drugs. Practical Clinical Journal of Integrated Traditional Chinese and Western Medicine 2014;14:90-1.

40 Guo F. Pharmacy intervention impact analysis on the use of quinolones. Journal of Clinical Medical Literature 2015;2:12-13.

41 Zhou Y, Ma L-Y, Zhao X, et al. Impact of pharmacist intervention on antibiotic use and prophylactic antibiotic use in urology clean operations. J Clin Pharm Ther 2015;40:404-8.

42 Li Z, Fang Z, Ge M, et al. Intervention effects of integrated pharmaceutical care for community chronic obstructive pulmonary disease patients. Chinese General Practice 2011;14:1496-500.

43 Chen $\mathrm{Y}$. Economic value of pharmaceutical intervention in the treatment of hypertension in community. China Journal of Pharmaceutical Economics 2016:12:11-14.

44 Huang S, Hu W. Effect of pharmaceutical care on rational drug use in hospitalized hypertensive patients. Journal of Community Medicine 2013;11:85-6.
45 Chen L, Su Y. Impact on the clinical efficacy of elderly patients with chronic obstructive pulmonary disease pharmacy services. Int $J$ Respir 2016;36:1244-7.

46 Wu M, Lv H, Yang H, et al. The Value of Pharmacy Intervention on Medication of Diabetic Patients with Hypertension[J]. J Med Theor \& Prac 2016;29:3175-7.

47 Jiang J, Shi X, Zhang Y, et al. Comparative study on effects of pharmaceutical intervention on clinical therapy for pulmonary tuberculosis patients complicated with diabetes mellitus. Chin J Mod Appl Pharm 2017;34:288-92.

48 Xin C, Ge X, Zheng L, et al. Evaluation of pharmaceutical care in a diabetes ward from China: a pre-and post-intervention study. Int $J$ Clin Pharm 2013;38:1-7.

49 Xin C, Ge X, Yang X, et al. The impact of pharmaceutical care on improving outcomes in patients with type 2 diabetes mellitus from China: a pre- and postintervention study. Int J Clin Pharm 2014;36:963-8.

50 Long E-W, Hu M, Tong R-S, et al. Analysis on the cost-effectiveness of pharmaceutical care for type II diabetic outpatients. Chin Hosp Pharm J 2014;34:139-42.

51 Chen Y-B, Zhao J. Cost-Effectiveness ratio of the pharmaceutical intervention in the treatment of patients with diabetes mellitus in the community. Chinese Journal of New Clinical Medicine 2014:7:343-5.

52 Li Z, Wang L, Shi Z, et al. Pharmaceutical Care Pathway for Rheumatic Mitral Stenosis With Atrial Fibrillation[J]. Chinese General Practice 2015;18:1317-21.

53 Wang S. Pharmaceutical care in the clinical pathway of left atrioventricular stenosis and atrial fibrillation in rheumatic heart disease. For all health 2016;10.

54 Qi P, Yuan Y, Li P, et al. The role of pharmaceutical care for clinical pharmacists during the treatment of patients with severe craniocerebral injury. Contemporary Medicine 2016;22:139-40.

55 Han F, Li Z, Yang W. Effects of clinical pharmacists participating in the implementation of femoral neck fracture clinical pathway under DRGs-PPS. China Pharmacy 2017;28:3281-4.

56 Liu M, Zhu X, Chen Z, et al. Analysis of clinical effect of involvement of clinical pharmacists in perioperative total parenteral nutrition prescription for cancer patients. Chinese Journal of Clinical Nutrition 2016;24:364-8.

57 Zhang C, Zhang L, Huang L, et al. Clinical pharmacists on medical care of pediatric inpatients: a single-center randomized controlled trial. PLoS One 2012;7:e30856.

58 Jiang S-P, Zheng X, Li X, et al. Effectiveness of pharmaceutical care in an intensive care unit from China. Saudi Med J 2012;33:756-62.

59 Committee for pharmacy administration, Chinese hospital association. Summary report for clinical pharmacist pilot training project, 2009. Available: http://www.chinadtc.org.cn/a/ linchuangyaoshi/guizhangzhidu/2013/0411/267.html [Accessed 26 Dec 2018].

60 Drummond MF, Jefferson TO. Guidelines for authors and peer reviewers of economic submissions to the BMJ. BMJ 1996;313:275-83. 